\begin{tabular}{cc}
\hline Revista de & Journal of Integrated \\
GESTÃO COSTEIRA Integrada COSTALZONE MANAGEMENT \\
\hline
\end{tabular}

\title{
Situação ecológica, socioeconómica e de governança após a implementação do primeiro plano de ordenamento no Parque Marinho Professor Luiz Saldanha (Arrábida, Portugal): II - percepções de utentes
}

\author{
Yorgos Stratoudakis@, , ; Flavio Fernández ; Miguel Henriques ${ }^{\mathrm{b}}$; João Martinss ${ }^{\mathrm{b}}$, Rogélia Martins ${ }^{\mathrm{a}}$
}

\begin{abstract}
Resumo
O Parque Marinho Professor Luiz Saldanha (PMLS) foi criado em 1998 e está regulado por um plano de ordenamento desde 2005. Este estudo apresenta os resultados de um inquérito que recolheu percepções de utentes do PMLS sobre a situação ecológica, socioeconómica e de governança 4-7 anos após a implementação faseada das regras nesta área marinha protegida (AMP). As 37 perguntas sobre o sistema social-ecológico e algumas perguntas auxiliares foram respondidas por 64 utentes do PMLS divididos em seis categorias alvo: pescadores profissionais a operar fora do PMLS, pescadores lúdicos, mergulhadores, investigadores, elementos da administração e outra. Diferente estratégia de amostragem foi seguida para cada categoria de utentes, em função do conhecimento prévio de indivíduos com elevado nível de familiaridade com os assuntos do inquérito. Foram respondidas 1694 das 2368 perguntas colocadas $(71.5 \%$ de resposta, com ausência de resposta na grande maioria dos casos por desconhecimento). As respostas agregadas foram utilizadas para formar indicadores de tendência para assuntos relevantes para qualquer AMP e comparadas estatisticamente com uma distribuição de respostas teórica normal, centrada na percepção de ausência de mudança. Foi também considerado o efeito da opinião pessoal do inquirido sobre o PMLS, avaliando a existência de diferenças significativas na distribuição das respostas de "optimistas" (inquiridos cuja aceitação do PMLS melhorou nos últimos anos) versus restantes. No geral, das respostas dos utentes sobressai uma visão positiva para a evolução do PMLS e da sua interacção com a pesca até 2012, com a excepção de alguns aspectos socioeconómicos importantes ligados à pesca. Existe também alguma variabilidade de perspectivas entre categorias (nomeadamente para assuntos ecológicos) e foram detectados seis casos de diferenças significativas na distribuição de respostas entre "optimistas" e restantes. Comparando estes resultados com os resultados do inquérito para os pescadores do PMLS (Stratoudakis et al., 2015), denota-se a prevalência de uma divergência de percepções nos assuntos ecológicos (com os pescadores a não detectar melhorias) e de uma convergência nos assuntos socioeconómicos e de governança. O objectivo conjunto destes trabalhos é apoiar a avaliação da eficácia de gestão no processo de revisão do plano de ordenamento do PMLS e a iniciação de um plano de monitorização longitudinal focado em metodologias participativas.
\end{abstract}

Palavras-chave: AMP, Sistema Social-Ecológico, Monitorização Participativa, Questionário, Avaliação de Eficácia de Gestão

\footnotetext{
(a) Corresponding author, to whom correspondence should be addressed. e-mail: <yorgos@ipma.pt>

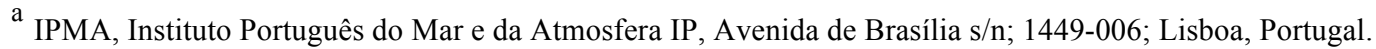

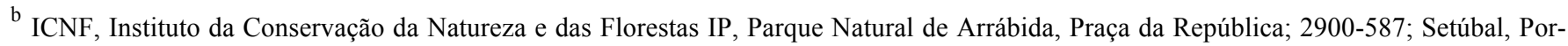
tugal.
}

Submission: 23 DEC 2014; Peer review: 8 FEB 2015; Revised: 18 FEB 2015; Accepted: 23 FEB 2015; Available on-line: 25 FEB 2015

This article contains supporting information online at http://www.aprh.pt/rgci/pdf/rgci-572 Stratoudakis Supporting-Information.pdf
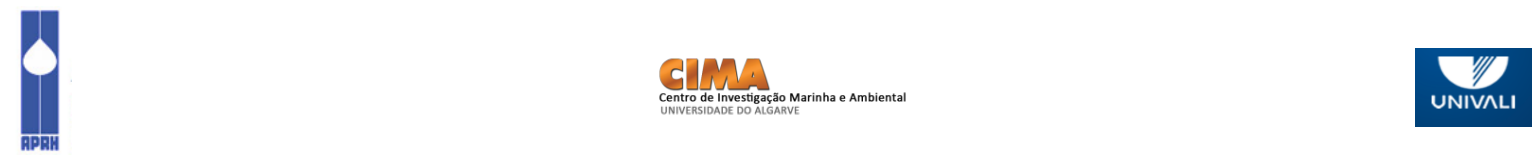


\section{Abstract}

Ecological, socioeconomic and governance conditions after the implementation of the first spatial plan in the Prof. Luiz Saldanha Marine Park (Arrábida, Portugal): II-user perceptions

The Prof. Luiz Saldanha Marine Park (PMLS - Arrábida, Portugal) was created in 1998 and has been managed according to a spatial plan since 2005. This study presents the results from an inquiry on the perceptions of PMLS users in relation to the ecological, socioeconomic and governance situation in the Park 4-7 years after the phased implementation of spatial rules in this Marine Protected Area (MPA). The 37 questions on the social-ecological system and some auxiliary questions were answered by 64 PMLS users from six categories: fishers operating outside the MPA, recreational fishers, divers, researchers, public administration and others. A distinct sampling strategy was followed for each user category depending on the level of previous knowledge of people with high level of familiarity with the questionnaire issues (close to a census on researchers and public administration members with specific activity in PMLS; snowball sampling for professional and recreational fishers; and provision of contacts of experienced divers from the Portuguese Centre of Sub-aquatic Activities). Respondents were asked to indicate their perception of improvement (moderate or large), stability or deterioration (moderate or large) on an ordinal scale of possible responses (5 levels Likert-type scale) for each of the issues at question. In total, 1694 replies were obtained to the 2368 questions placed (response rate of $71.5 \%$, with the great majority of non-response being due to lack of knowledge). Aggregated replies were used to construct trend indicators on issues relevant for any MPA and were compared statistically with a series of samples from a theoretical distribution of replies centered in the lack of change (standardized normal distribution, truncated at 2 standard deviations). The effect of respondent opinion on the PMLS was tested by comparing the distribution of replies between "optimists" (people whose opinion of the PMLS improved in the last 4-5 years) and others. Overall, user replies indicate a positive view on the evolution of the PMLS and its interaction with small scale fisheries until 2012, with 2.04 replies of perceived improvement for every reply of perceived deterioration. This ratio was substantially lower for socioeconomic issues, where a perceived significant improvement in PMLS knowledge and its dissemination were counterbalanced by a perceived deterioration in the ability of excluded fishers to maintain a living related to fishing and the attraction of fishing as employment for youth. This contrast is also detected when comparing the distribution of replies to 5 socioeconomic and governance questions identically phrased with respect to fishers and other users: on two issues (earnings and management solutions) the same group of respondents considered that the evolution in the last 4-5 years was significantly better for other users than for fishers (although no significant difference was found in the evolution of group conflicts with the MPA managing authority). Variation in replies was detected among user categories (mainly in ecological issues), while significant differences between the replies of "optimists" and others were only found in 6 questions (PMLS acceptance by fishers, other users and local community; attraction of fishing as employment for youth; coverage by brown macroalgae and sightings of seabirds and marine mammals). Comparing these results with those of the inquiry to PMLS fishers (Stratoudakis et al., 2015) there is a prevalence of a divergence in perceptions for ecological issues (with PMLS fishers not detecting the improvements perceived by other users) and a convergence in socioeconomic and governance issues (both those indicating improvement and those indicating deterioration). The joint objective of these two studies is to inform the management effectiveness evaluation that is included in the process of revision of the spatial plan for the PMLS and to set the basis for a longitudinal monitoring plan focusing on participatory methods.

Keywords: MPA, Social-Ecological System, Participatory Monitoring, Questionnaire, Management Effectiveness Evaluation

\section{Introdução}

O Parque Marinho Professor Luiz Saldanha (PMLS Arrábida, Portugal) foi o primeiro Parque Marinho a ser criado em Portugal continental em 1998. O PMLS estende-se numa área de $53 \mathrm{~km}^{2}$ ao longo de $38 \mathrm{~km}$ de linha da costa da Península de Setúbal, desde os baixios da entrada do estuário do Sado - na praia da Figueirinha - até aos biótopos rochosos situados a norte do Cabo Espichel (Henriques et al., in press), com limite na praia da Foz. A sua maior parte é exposta a Sul, com a Serra de Arrábida a proteger dos ventos $\mathrm{N}$ e $\mathrm{NO}$, criando assim condições de reduzido hidrodinamismo durante longos períodos do ano. Esta extensão de costa abrigada e protegida da ondulação noroeste é rara na faixa atlântica da Península Ibérica, permitindo o desenvolvimento de comunidades ecológicas bastante estruturadas (Henriques et al., 2007, Henriques et al., 2013). O Parque é habitado por aproximadamente 1320 espécies marinhas (Cunha et al., 2014) e contém quatro tipos de habitats sensíveis: Bancos de areia permanentemente cobertos por água do mar pouco profunda; Grutas marinhas submersas ou semi-submersas; Lodaçais e areias a descoberto na maré baixa; e Recifes rochosos. Parte do PMLS foi também designada como Zona de Protecção Especial (ZPE), fazendo assim parte da Rede Natura 2000 desde 2003.

Para além da elevada importância da zona na actividade de pesca profissional (ver Stratoudakis et al., 2015), a costa da Arrábida tem sido também nas décadas recentes lugar privilegiado para actividades lúdicas ligadas ao mar, nomeadamente mergulho com escafandro autónomo, pesca lúdica apeada e embarcada, náutica de recreio, etc. (Cabral et al., 2008). A regulamentação inicial do PMLS em 1998 baniu da área do Parque a pesca da ganchorra e a apanha de bivalves com escafandro autónomo, enquanto que o POPNA proibiu também a pesca do cerco, a pesca submarina de apneia e a aquicultura. O zonamento do PMLS pelo 
POPNA (ver Informação de Suporte - Figura IdS 1) também condicionou as restantes actividades lúdicas, excluindo-as da zona de reserva de protecção total, proibindo a fundeação de embarcações junto à costa nas áreas de protecção parcial e restringindo a pesca lúdica apeada e embarcada nas zonas de protecção complementar. Estes condicionalismos, juntamente com o modo atribulado que se desencadeou o processo de consulta pública antes da entrada em vigor do POPNA (Carneiro, 2011; Vasconcelos et al., 2012), criaram insatisfação em vários grupos de utentes que, sem estar contra a criação da AMP, se declararam contra as medidas do POPNA para a sua actividade (ver Cabral et al., 2008 para respostas de pescadores lúdicos e praticantes de náutica de recreio).

O questionário utilizado neste estudo foi desenvolvido para caracterizar os sistemas social-ecológicos complexos que surgem da interacção da pequena pesca com as AMPs (ver Stratoudakis et al., 2015). Os temas do inquérito são divididos entre assuntos ecológicos, socioeconómicos e de governança, com indicadores específicos para avaliar o estado actual (principalmente no inquérito para os pescadores) ou a tendência recente. O exercício foi desenvolvido através de inquéritos e inclui as percepções de outros utentes do PMLS (presente trabalho), para além do registo de informações e opiniões dos pescadores com licença de pescar no PMLS sobre os mesmos temas (Stratoudakis et al., 2015). O objectivo geral do conjunto destes trabalhos é apoiar a avaliação da eficácia de gestão no processo da primeira revisão do plano de ordenamento do PMLS, focado em metodologias participativas (por isto, ambos os documentos estão escritos em Português e submetidos numa revista com livre acesso on-line).

\section{Materiais e métodos}

\section{i) Inquérito}

O questionário utilizado neste estudo foi desenvolvido no âmbito do projecto MAIA (Áreas Marinhas Protegidas no arco Atlântico; http://www.maianetwork.org) para ser aplicado em várias AMPs em paralelo com um questionário dirigido aos pescadores com licença de pesca na AMP (ver Stratoudakis et al., 2015). No caso do inquérito para os utentes que não pescadores do PMLS, no total foram desenvolvidas 11 perguntas ecológicas, 11 socioeconómicas e 15 de governança (Pomeroy et al., 2004), identificando indicadores apropriados para avaliar a tendência de cada propriedade do sistema comparativamente à sua situação 4-5 anos antes (nesta primeira aplicação, aproximadamente o período desde a entrada em pleno funcionamento dos condicionalismos espaciais do plano de ordenamento). As perguntas foram desenhadas para registar directamente a percepção dos inquiridos sobre a tendência de cada indicador numa escala Likert de cinco níveis ordinais (Norman, 2010). Estes níveis foram centrados na percepção de ausência de mudança, considerando dois cenários de deterioração (deteriorou ou deteriorou muito) e dois de melhoria (melhorou ou melhorou muito) no período de referência. Por exemplo, para avaliar a percepção dos inquiridos sobre a propriedade socioeconómica ligada às condições de vida dos pescadores da AMP foi elaborada a pergunta: "Na comunidade de Sesimbra ligada ao PMLS, considera que durante os últimos 4-5 anos o rendimento dos pescadores em relação ao ordenado mínimo nacional: decresceu muito; decresceu; manteve-se; aumentou; aumentou muito; não sei; não respondo" e foi comparada com uma pergunta idêntica para os rendimentos dos restantes utentes da AMP.

Para além destas 37 perguntas na escala Likert, foram incluídas no questionário algumas perguntas sobre o inquirido e a clareza e possível utilidade do questionário. Todas as perguntas foram inicialmente desenvolvidas em inglês, no contexto da colaboração de equipa internacional no âmbito do projecto MAIA, e depois foram traduzidas em português para aplicar no PMLS. Na ficha do questionário um preambulo introduziu os objectivos e componentes do estudo, garantindo o anonimato aos inquiridos. As perguntas foram também transcritas num questionário on-line (software Surveymonkey) para fornecer a possibilidade de preenchimento remoto a inquiridos que podiam ser contactados electronicamente. Cópias do questionário em papel foram entregues aos inquiridos com indisponibilidade para responderem no momento da entrevista e sem facilidade para preencher o inquérito on-line.

\section{ii) Amostragem}

O questionário dos utentes foi desenhado para ser respondido por pessoas com elevada probabilidade de algum conhecimento específico sobre a interacção pequena pesca-PMLS. Com base na experiência anterior sobre o sistema (contacto directo com vários utentes e participação em sessões públicas sobre a problemática do PMLS - Vasconcelos et al., 2012), foram identificadas cinco categorias específicas de utentes com acção reconhecida no contexto do PMLS:

- administração pública (incluindo a entidade gestora do PMLS, a Câmara local, a Capitania e a administração pesqueira);

- investigadores (só incluindo investigadores com trabalho desenvolvido nesta zona);

- pescadores lúdicos;

- mergulhadores (com experiência no PMLS);

- pescadores profissionais que desenvolvem a sua actividade fora do PMLS (mas a operar do porto de Sesimbra);

- outros. 
A sexta categoria (Outros) foi criada para inquiridos que não se sentiam suficientemente representados por nenhuma das categorias anteriores. Optou-se por não chamar esta última categoria comunidade local, uma vez que isto pressupunha uma abordagem diferente (querer saber a opinião da comunidade independentemente do grau de contacto com o PMLS) e um desenho de amostragem adequado para obter uma amostra representativa da população geral da zona do Parque.

O inquérito foi efectuado no verão de 2012 (JulhoSetembro), simultaneamente com o inquérito dirigido aos pescadores do PMLS. Dentro de cada categoria procurou-se amostrar um painel de "peritos" (Davis and Wagner, 2003), seguindo distintas estratégias de amostragem em diferentes categorias mas procurando manter o tamanho da amostra semelhante ou igual: para pescadores com conhecimento do PMLS mas a operar fora do Parque e para pescadores lúdicos, foi seguida a metodologia de amostragem de bola de neve - durante as entrevistas com os pescadores profissionais com licença do PMLS procurou-se obter o contacto de alguns elementos iniciais e através do contacto com os próprios foram depois obtidos contactos adicionais; para os investigadores, a administração pública e os outros o contacto baseou-se em conhecimento prévio (incluindo a experiencia durante o projecto MARGOV - Vasconcelos et al., 2012); finalmente, para identificar o grupo de mergulhadores, foi pedido auxílio à direcção do CPAS (Centro Português de Actividades Subaquáticas) que forneceu uma lista de contactos eletrónicos dos mergulhadores com maior experiencia no PMLS e incentivou a sua participação. $O$ objectivo inicial era obter 15 respostas por categoria, tendo em conta que a população total da vila de Sesimbra ronda os 5000 habitantes e que nas sessões públicas mais participadas sobre o PMLS (projecto MARGOV - Vasconcelos et al., 2012) raramente participaram mais que 70-80 pessoas.

O inquérito foi respondido por 64 pessoas (ver Informação de Suporte - Respostas ao inquérito e Tabela IdS 1). A idade dos inquiridos variou entre os 19 e os 80 anos (ver Informação de Suporte - Figura IdS 2), com a média da amostra situada aos 46 anos e com as mulheres em média ligeiramente mais novas que os homens (43 vs 47 anos). A grande maioria dos inquiridos tinha mais que 10 anos de contacto com o sistema, sendo este período em média mais longo para os pescadores profissionais a operar fora do PMLS (categoria com maior idade média) e mais curto para administração pública e investigadores.

Foram respondidas 1694 das 2368 perguntas colocadas (37 perguntas a 64 inquiridos), numa percentagem global de resposta de $71,5 \%$. Na grande maioria dos casos a ausência de resposta era por desconhecimento, sendo que a falta de vontade de comunicar uma opinião pessoal só foi invocada em 3 situações relacionadas com a aceitação pessoal do PMLS. A taxa de resposta foi ligeiramente menor para as perguntas ecológicas $(69,7 \%)$, para as quais pescadores, pescadores lúdicos e mergulhadores tiveram uma taxa de resposta muito superior à das outras três categorias. Globalmente a categoria com maior taxa de resposta foi a dos pescadores lúdicos $(86 \%)$ e com menor a dos mergulhadores $(56 \%)$, que tiveram relativamente baixas taxas de resposta para perguntas socioeconómicas e de governança. A pergunta mais respondida foi sobre a aceitação do PMLS pelo inquirido (que só não foi respondida por 3 pessoas que não quiseram emitir opinião) e a menos respondida foi sobre a participação dos pescadores nas deliberações da Associação (que não foi respondida por 37 pessoas).

\section{iii) Exploração e análise de dados}

Os resultados do questionário foram codificados, explorados e analisados utilizando o software R (versão $3.1-R$ Core team 2014). O caracter ordinal das respostas na escala Likert foi mantido numa escala numérica natural, na qual a resposta de ausência de mudança foi codificada como zero. Assim, respostas de melhoria e grande melhoria ficaram representadas por 1 e 2 unidades positivas respectivamente e respostas de deterioração e grande deterioração ficaram representadas por 1 e 2 unidades negativas respectivamente. Para cada inquirido, foi calculado o número total de respostas fornecidas por cada um dos três grupos de perguntas e para os 37 indicadores no total (i.e. excluindo não sei ou não respondo). Foi também calculado o número de respostas de percepção de melhoria (i.e. resposta positiva, independentemente da sua qualificação) e o número de respostas de percepção de deterioração (resposta negativa) para cada um dos três grupos de perguntas e para a totalidade. Nos casos que um inquirido tinha pelo menos uma resposta negativa, foi também calculado o ratio de respostas positivas/negativas para cada grupo de perguntas e para o inquérito na totalidade.

As respostas para cada pergunta foram sumarizadas em histogramas de densidades (ver Informação de Suporte Figuras IdS 3-5). Para facilitar a visualização dos padrões em cada resposta, a função de densidade de probabilidade da distribuição normal padrão $\sim \mathrm{N}(0,1)$ foi sobreposta em cada histograma. Para cada pergunta foram geradas 100 amostras aleatórias de uma distribuição normal padrão (usando como tamanho de amostra o número de respostas válidas da pergunta valor $n$ apresentado em cada histograma) que foram aproximadas ao número inteiro mais próximo. Os raros valores de $>2$ desvios padrão da média centrada nestas amostras aleatórias foram substituídos por 2 (o valor mais extremo considerado no inquérito). Cada uma destas 100 amostras geradas foi comparada com a dis- 
tribuição das respostas no inquérito através do teste Wilcoxon para duas amostras (Man-Whitney). O número de comparações para as quais a diferença na distribuição foi significativa (i.e. $\mathrm{p}<0.05$ ) foram registadas (estatístico $k$ expresso na forma de percentagem apresentado em cada histograma). Este teste estatístico fornece uma estimativa do grau de desvio no padrão de respostas numa pergunta em comparação a uma distribuição normal padrão truncada nos extremos e centrada na percepção de ausência de mudança. Apesar dos pressupostos implícitos nesta comparação (que a percepção sobre um indicador pode ter uma distribuição continua subjacente e que a transição entre níveis ordinais na escala de Likert pode ser definida com base no desvio padrão desta distribuição), esta abordagem permite a rápida visualização dos dados e facilita a interpretação dos resultados sem violar a natureza ordinal da informação-base.

Para além da anterior metodologia de avaliação da importância da alteração de percepção num indicador em função da percepção 4-5 anos antes, foi também investigada a coerência entre inquiridos, adoptando a estratégia seguinte: a análise exploratória dos dados mostrou que na pergunta sobre a aceitação pessoal do PMLS havia um claro efeito de anos de experiência no sistema (Figura 1), bastante correlacionado com a idade dos inquiridos e algo correlacionado com a categoria de utente (sendo que os 5 pescadores a operar fora do Parque eram também os que tinham maior idade média e mais anos de contacto com o PMLS). Foi também verificada uma forte ligação entre o nível de aceitação pessoal do PMLS e o ratio de respostas positivas/negativas na totalidade do inquérito, sendo ele substancialmente inferior (e abaixo de 1) para inquiridos cuja aceitação do PMLS deteriorou nos anos recentes.

Uma vez que os dados disponíveis não permitem uma clarificação entre o efeito categoria de utente, idade e anos de contacto com o PMLS, optou-se por considerar a importância do "optimismo" nos resultados para cada indicador. Assim, em cada pergunta foi também comparada a distribuição das respostas dos "optimistas" (i.e. que a sua aceitação melhorou nos últimos 4-5 anos) com os restantes para avaliar se a mensagem geral tinha suficiente concordância entre utentes com diferentes perspectivas sobre o PMLS. Foi utilizado de novo o
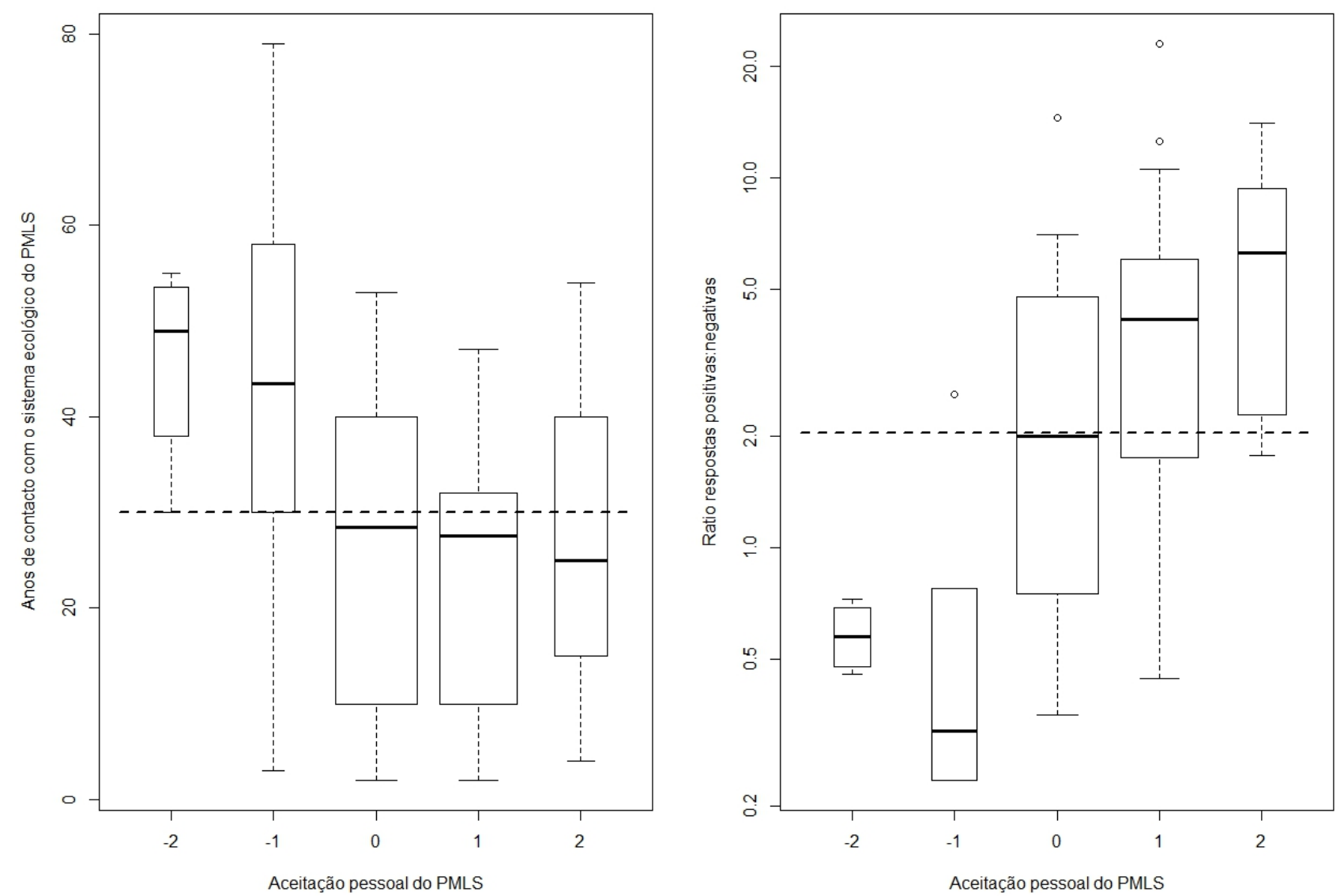

Figura 1 - Distribuição dos anos de contacto do inquirido com o sistema da AMP (gráfico de caixa e "bigodes" de esquerda) e do ratio de respostas positivas sobre negativas em todo o inquérito (gráfico de caixa e "bigodes" de direita) em função da resposta de tendência na aceitação pessoal da AMP de cada inquirido.

Figure 1 - Distribution of years of contact with the MPA system (box and whisker plots of left) and ratio of positive over negative answers in the entire questionnaire (box and whisker plots of right) as a function of the reply on trend of personal MPA acceptance by each respondent. 
teste Wilcoxon para duas amostras, esta vez corrigido para comparações múltiplas (nível nominal de rejeição da hipótese nula o 0.1 , que corrigido para 36 comparações corresponde a $p<0.0028$ em cada aplicação).

\section{Resultados}

\section{i) Indicadores ecológicos}

Para 8 das 11 perguntas ecológicas, a percepção modal é positiva (i.e. de alguma melhoria durante os últimos 4-5 anos) o que se reflecte também na soma das respostas ecológicas (ver Informação de suporte - Figura IdS 3). As únicas modas a situar-se na percepção de manutenção da situação anterior são os casos das macroalgas castanhas (indicador sobre estado de habitats) e os avistamentos de aves e mamíferos marinhos (indicador sobre presença de predadores de topo na comunidade). No caso das macroalgas verdes prevalece também a percepção da manutenção da situação, mas neste caso a resposta pode ser considerada positiva, uma vez que não aponta para aumentos da eutrofização na zona do PMLS.

Apesar da prevalência de modas com percepções positivas, a comparação com amostras aleatórias provenientes de uma distribuição normal padrão só indicam forte probabilidade de resultados significativos no caso da abundância de peixes juvenis (estatístico $k>95 \%$, i.e. o teste Wilcoxon detectou uma diferença significativa com a distribuição das respostas dos inquiridos em 96 das 100 comparações com as amostras aleatórias da distribuição normal padrão geradas). Esta diferença é suportada pela densidade desproporcionalmente elevada de inquiridos que consideraram que a abundância de peixes juvenis tinha aumentado muito nos anos recentes. Existe também uma probabilidade relativamente elevada de diferença significativa para a percepção da presença de peixes estranhos ou raros e para o esforço de pesca na fronteira do PMLS. Nestes casos a moda positiva aproxima ou ultrapassa a metade da amostra, mas diferenças significativas no teste Wilcoxon são só detectadas em $91 \%$ e $82 \%$ das comparações respectivamente.

Considerando a coerência das respostas ecológicas entre "optimistas" e restantes, não existe nenhuma pergunta com diferenças significativas na distribuição das respostas após a correcção para múltiplos testes. Todavia, no caso da pergunta sobre a cobertura com algas castanhas $(W=95,5 ; p=0,0046)$ e na pergunta sobre o avistamento de aves e mamíferos $(W=155$; $p=0,0066$ ) existe uma diferença significativa para um nível de confiança ligeiramente inferior. Nestes casos, a percepção modal dos "optimistas" aponta para alguma melhoria nos últimos anos, uma minoria acha que a situação melhorou muito e quase ninguém acha que a situação piorou, enquanto que a moda para os restantes está na ausência de mudança, com alguns a considerar que a situação deteriorou muito e quase ninguém a achar que a situação melhorou (ver Figura 2)

\section{ii) Indicadores socioeconómicos e de governança}

Ao contrário das perguntas ecológicas, existe maior variabilidade no posicionamento das modas entre perguntas socioeconómicas, mas também menor discrepância entre as opiniões dos inquiridos optimistas e restantes (ver Informação de Suporte - Figura IdS 4). Assim, modas negativas prevalecem no caso da probabilidade dos pescadores excluídos do PMLS continuarem a ganhar a vida através da pesca e na atractividade da pesca no PMLS como profissão para jovens. Por outro lado, existem quatro perguntas com a moda a indicar uma percepção de melhoria ou aumento (no caso dos rendimentos de outros utentes, da criação e divulgação de conhecimento sobre o PMLS e da procura de licença para pescar no PMLS). Para as restantes cinco perguntas a moda está situada na percepção de ausência de mudança, apesar de - no caso dos rendimentos dos pescadores do PMLS e dos seus conflitos com pescadores lúdicos - a frequência de respostas com percepção de alguma deterioração ser quase igual à frequência modal.

A comparação com as amostras aleatórias provenientes da distribuição normal padrão denota uma probabilidade muito elevada de diferenças significativas no caso da probabilidade dos pescadores excluídos continuarem a viver da pesca (i.e. deterioração significativa) e nos casos da criação e divulgação do conhecimento sobre o PMLS (i.e. melhoria significativa). Considerando a coerência das respostas socioeconómicas entre "optimistas" e restantes, a única diferença significativa surge para a atractividade da pesca no PMLS para jovens ( $W=136,5 ; p=0,0022)$ : para os "optimistas" a moda está situada num aumento desta atractividade nos últimos 4-5 anos, enquanto que para os restantes está numa deterioração (i.e. modas parcelares com 2 níveis ordinais de diferença - ver Fig. 2). No total, a percepção globalmente neutra representada pela soma dos indicadores socioeconómicos é algo enganadora, uma vez que está a equilibrar percepções globalmente negativas para indicadores socioeconómicos ligados directamente aos pescadores com percepções globalmente positivas para indicadores mais relacionados com outros utentes ou a comunidade geral. Estas percepções são no geral partilhadas entre categorias, apesar de no caso da atractividade para jovens existir uma separação significativa baseada no grau de aceitação do PMLS.

Ao contrário das perguntas socioeconómicas, no caso de governança não houve perguntas com moda na resposta negativa (ver Informação de suporte - Figura IdS 5). Em 7 das 15 perguntas a moda está situada na per- 

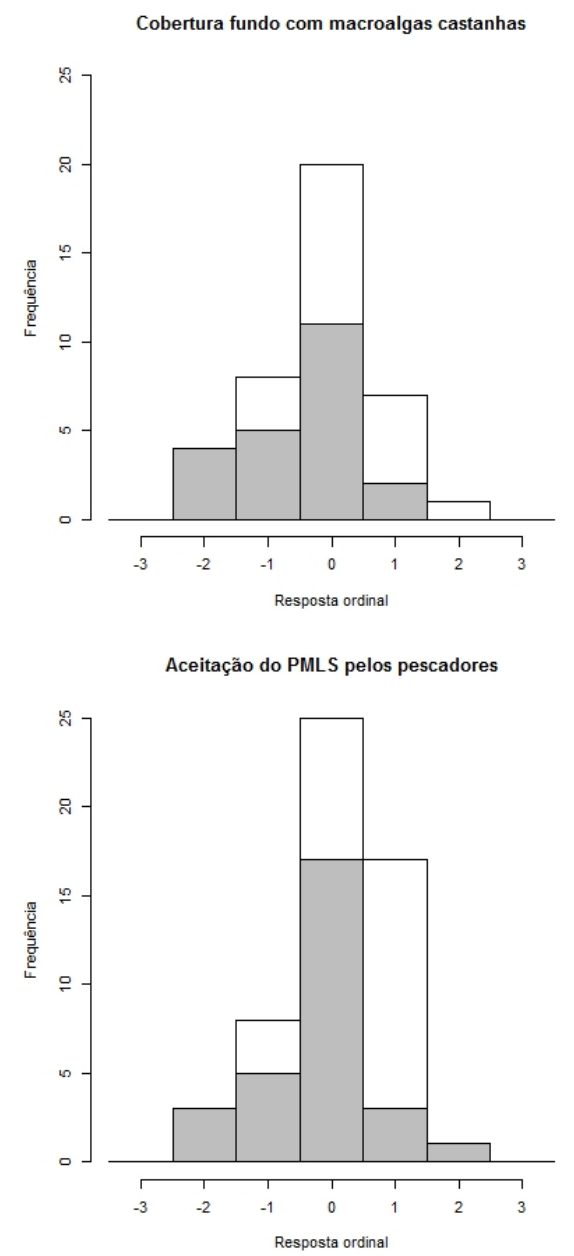

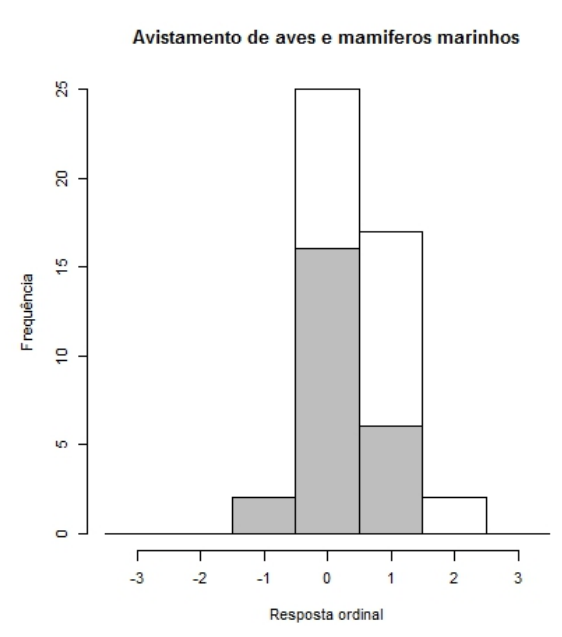

Aceitação do PMLS pelos outros utentes

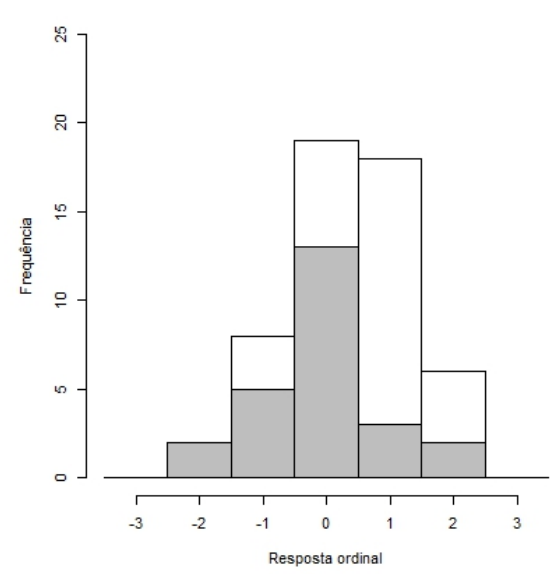

Atractividade da pesca no PMLS como profissão para jovens

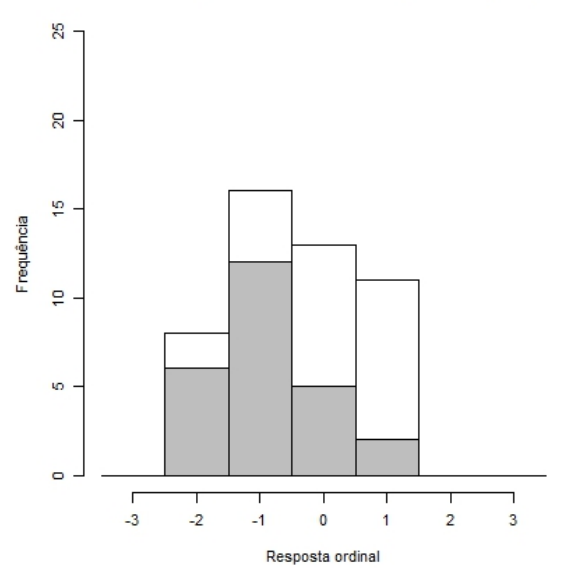

Aceitação do PMLS pela comunidade local

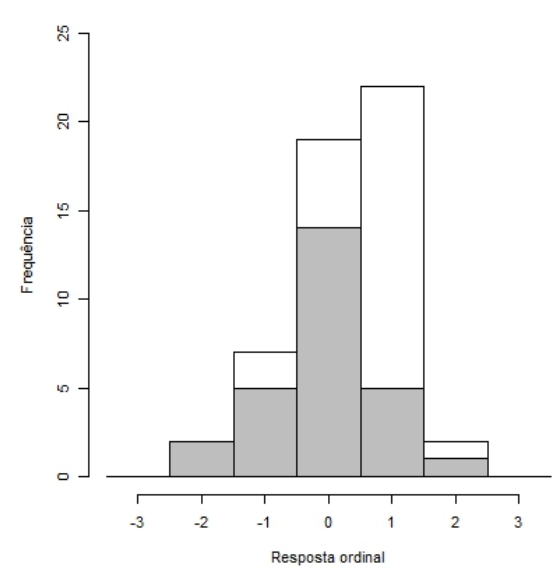

Figura 2 - Histogramas para perguntas com diferenças significativas na distribuição de respostas entre "optimistas" (parte da barra em branco) e restantes (parte da barra em cinzento).

Figure 2 - Histograms for questions with significant differences in the distribution of answers from "optimists" (white part of bar) and others (grey part of bar).

cepção de ausência de mudança, incluindo as três perguntas sobre a aceitação do PMLS pelos utentes e os próprios inquiridos. Modas positivas foram observadas nas três perguntas relacionadas com a legitimação dos pescadores e dos seus representantes nos processos de decisão sobre o PMLS, nas perguntas do cumprimento das regras do PMLS (mas não no cumprimento das regras gerais de pesca), no encontro de soluções de gestão para outros utentes do PMLS e na aceitação do PMLS pela comunidade local e as comunidades vizinhas.

Das perguntas com moda positiva, só a da capacidade de intervenção da Associação dos pescadores mostra um resultado altamente significativo quando comparada com as amostras aleatórias da distribuição normal padrão. Todavia, a pergunta sobre o número de pescadores associados é a única pergunta de governança com uma distribuição bimodal na frequência das respostas (e juntamente com a cobertura de macroalgas castanhas as únicas a mostrar alguma bimodalidade em todo o inquérito), indicando que existe também uma minoria de inquiridos a achar que o associativismo diminuiu nos últimos 4-5 anos. Uma vez que os dados da AAPCS apontam para um aumento do número de associados nestes anos, esta bimodalidade pode estar relacionada com a diferença de perspectivas entre pescadores de aiolas (cuja representação pela AAPCS é parcial, havendo muitos não associados em nenhuma organização) e embarcações maiores (cuja representação é total para os botes com licença do PMLS e muito elevada para embarcações maiores).

Finalmente, na comparação entre "optimistas" e restantes, verificou-se uma divergência significativa nas respostas sobre a aceitação do PMLS pelos pescadores ( $W=180,5 ; p=0,0027)$, pelos outros utentes $(W=175,5$; $p=0,0020)$ e pela comunidade de Sesimbra $(W=157$; $p=0,0008$ ). Nestes três casos (ver Fig. 2) existe uma clara diferença na moda, com os "optimistas" a apontar maioritariamente para alguma melhoria na aceitação do PMLS pelos pescadores, outros utentes e a comunidade geral, enquanto que os restantes estão centrados na ausência de mudança de opinião. Existem também diferenças na frequência dos que acham que a aceitação diminuiu muito, com nenhum dos "optimistas" a achar 
que a opinião dos intervenientes no PMLS piorou muito, enquanto que para os restantes existe uma minoria a acreditar nisto.

\section{iii) Comparações entre grupos}

No inquérito existem cinco perguntas sobre aspectos socioeconómicos e de governança formuladas de forma idêntica com respeito a pescadores e outros utentes, permitindo avaliar se os inquiridos têm uma perspectiva de evolução distinta para estes grupos desde a implementação do POPNA. Da comparação das respostas (Tabela 1), verifica-se que os inquiridos identificam diferenças significativas nas soluções de gestão e no rendimento: em ambos os casos é considerado que a situação dos outros utentes é comparativamente melhor que a dos pescadores, com um aumento nas soluções de gestão e nos rendimentos desde a implementação do PMLS para os outros utentes que não é observado para os pescadores do PMLS. Por outro lado, não são detectadas diferenças significativas entre estes dois grupos no nível de conflitualidade com a entidade gestora, na conformidade com as regras do PMLS ou na aceitação do PMLS (apesar que neste último caso o resultado é só marginalmente não significativo).

A Figura 3 fornece uma imagem sinóptica dos resultados deste inquérito e das variações de opinião entre temas e categorias de utentes, contabilizando o ratio de respostas positivas sobre negativas para perguntas ecológicas, socioeconómicas, de governança e total. Globalmente, as respostas positivas ultrapassam as respostas negativas num ratio de 2,04:1 (i.e. 1 resposta negativa ou muito negativa para cada 2.04 respostas positivas ou muito positivas nas 1119 respostas que identificaram alguma mudança da situação entre as 1694 perguntas respondidas pelos 64 inquiridos). Este padrão é verificado em todas as categorias de utentes com excepção dos pescadores profissionais a operar fora do PMLS, que forneceram mais respostas nega- tivas do que positivas para todos os tipos de perguntas. Para os restantes, o grau de optimismo varia, com a categoria não especifica (outros) e os pescadores lúdicos a terem ratios globais muito perto de $1 \mathrm{e}$ a administração e investigação a terem ratios globais substancialmente mais elevados. $\mathrm{O}$ ratio de respostas positivas é mais elevado para perguntas ecológicas $(3,01)$, intermédio para perguntas de governança $(2,31)$ e mais baixo para perguntas socioeconómicas $(1,23)$, nas quais para além dos pescadores, os pescadores lúdicos e os outros também fornecem mais respostas negativas do que positivas.

\section{iv) Comparações com o inquérito dos pescadores}

Para além das comparações anteriores, intrínsecas ao inquérito para outros utentes, existe também a possibilidade de comparar estes resultados com os principais resultados do inquérito para pescadores do PMLS (ver Stratoudakis et al., 2015). Esta comparação na maioria dos casos não pode ser directa (uma vez que as perguntas aos pescadores foram codificadas de forma a basearem-se na sua actividade) e em alguns casos nem sequer é possível (em situações que para os pescadores só existe um indicador de estado que nesta primeira aplicação não pode ser comparado com os indicadores de tendência do questionário para outros utentes). Todavia, para seis indicadores de tendência (ver Fig. IdS 4.1 em Stratoudakis et al., 2015) é possível comparar a distribuição das respostas dos pescadores com as dos outros utentes e avaliar a similitude das respostas dos dois grupos.

Para os assuntos ecológicos, a observação genérica mais marcante é da discrepância entre a percepção genericamente optimista dos outros utentes (com modas na percepção de alguma melhoria para a maioria das variáveis ecológicas, apesar de muitas delas não significativas) e a percepção de falta de mudança ou deterioração não significativa para os pescadores do PMLS.

Tabela 1 - Comparação da distribuição de respostas dos inquiridos às mesmas perguntas de cariz socioeconómica ou de governança com respeito aos pescadores ou aos outros utentes do PMLS. A hipótese nula é falta de diferença na resposta média para os dois grupos (Wilcoxon rank sum teste emparelhado). Graus de liberdade ( $d f$ ) para o teste correspondem ao número de inquiridos que respondeu cada pergunta para os dois grupos (pescadores e outros utentes).

Table 1 - Comparison of the distribution of replies to the same socioeconomic and governance questions with respect to fishers and other PMLS users. The null hypothesis is of lack of difference in the mean reply for the two groups (Wilcoxon paired rank sum test). Degrees of freedom (df) for the test correspond to the number of respondents that answered each question for both groups (fishers and other users).

\begin{tabular}{|l|c|c|c|}
\hline \multicolumn{1}{|c|}{ Assunto } & V & df & p \\
\hline Conformidade com regras PMLS & 116.5 & 49 & $0.167(\mathrm{~ns})$ \\
\hline Soluções de gestão & 14 & 36 & $0.008(* *)$ \\
\hline Conflitos com entidade gestora PMLS & 35 & 37 & $0.437(\mathrm{~ns})$ \\
\hline Aceitação PMLS & 15 & 46 & $0.053(\mathrm{~ns})$ \\
\hline Rendimento & 5.5 & 31 & $<0.001(* * *)$ \\
\hline
\end{tabular}




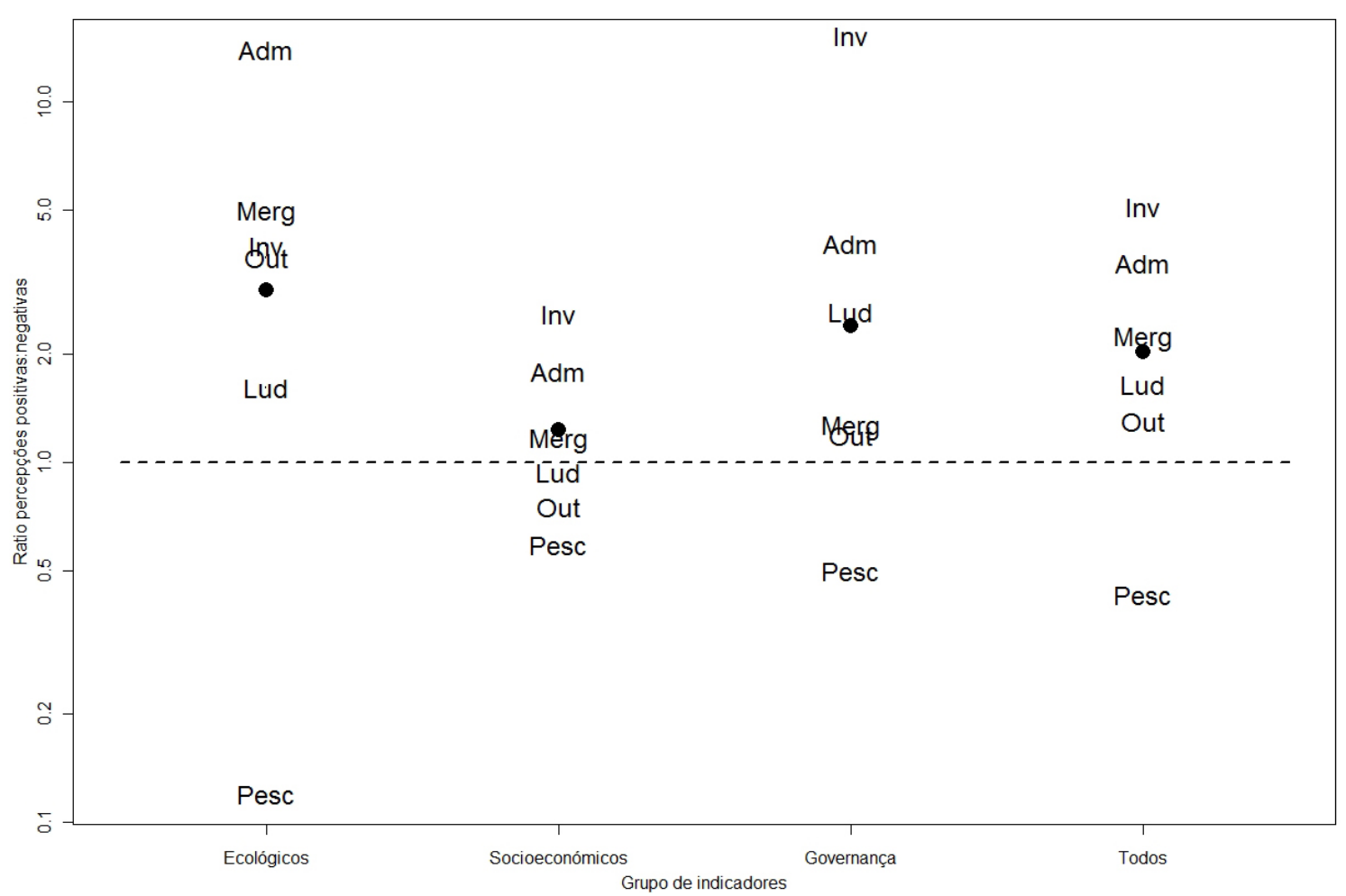

Figura 3 - Ratio de soma de respostas positivas sobre respostas negativas para cada grupo de perguntas (ecológicas, socioeconómicas, de governança e totais) e para cada categoria de inquiridos (legenda no símbolo). Ciclos pretos indicam média para cada grupo de perguntas entre categorias de utentes.

Figure 3 - Ratio of the sum of positive over negative answers for each group of questions (ecological, socio-economic, governance or total) and each category of stakeholders (symbol legend). Black circles indicate mean group value for all stakeholder categories.

Isto é confirmado pela diferença significativa na distribuição das respostas para quatro dos cinco indicadores de tendência ecológicos passíveis de comparar: a abundância de espécies alvo $(W=775 ; p=0,0010)$; a abundância de juvenis ( $W=256,5 ; p=0,0005)$; o número de espécies sem interesse comercial ( $W=409,5$; $p=0,0004)$; e a presença de peixes grandes $(W=660,5$; $p=0,0007)$. Em todos os casos a percepção maioritária dos outros utentes é de alguma melhoria nos últimos 45 anos, enquanto que para os pescadores estas variáveis ficaram inalteradas ou eventualmente diminuíram ligeiramente no caso da abundância das espécies alvo da pesca (ver Tabela IdS 4.1 em Stratoudakis et al., 2015).

A principal concordância ecológica nos dois inquéritos surge relacionada com o papel das macroalgas castanhas na diversidade e productividade do ecossistema na zona e as consequências do seu desaparecimento abrupto, assunto para o qual convergem as narrativas tanto na caracterização do ecossistema antes e depois, como no período de transição (fim dos anos 80/inicio dos anos 90) e as causas prováveis (varias hipóteses de "poluição"). Outro assunto de possível concordância é sobre o registo de algum aumento na observação de espécies estranhas ou raras nos últimos anos (percepção significativa para outros utentes) que é corroborado pela lista de espécies sem interesse comercial, raras ou estranhas registadas pelos pescadores. Também para o indicador de tendência ecológica relacionado com a presença de esforço de pesca nos limites do PMLS a comparação da distribuição das respostas não foi significativa ( $W=347 ; p=0.066)$, apesar da moda dos pescadores estar situada na ausência da resposta e a dos outros utentes na de alguma melhoria (mas não significativamente diferente da ausência de mudança).

Para os assuntos socioeconómicos e de governança, a observação genérica mais marcante é a da consonância geral sobre as consequências da criação do Parque para os pescadores excluídos, as perspectivas futuras para a pesca e o relacionamento dos pescadores do PMLS com a entidade gestora. Há uma percepção predominante entre os outros utentes que a probabilidade dos pescadores excluídos pelo PMLS continuar a ganhar a vida pela pesca e a atratividade da pesca para os jovens terem diminuído significativamente. Isto é corroborado pelo número de pescadores identificados como tendo saído da pesca depois da exclusão pelo PMLS e pela muito reduzida proporção de actuais pescadores a sugerir a pesca como primeira opção para o futuro de jovens familiares. Todavia, a relação dos pescadores com a entidade gestora (ICNF) não é vista pelos outros utentes como tendo sofrido deterioração neste período. Isto é apoiado pelas respostas dos pescadores sobre a 
importância dos conflitos com a entidade gestora em relação a outros problemas e a claramente maioritária vontade deles de embarcar num processo de co-gestão da actividade pesqueira no PMLS com o ICNF.

Houve também assuntos socioeconómicos e de governança para os quais se notaram algumas discrepâncias entre a resposta predominante dos pescadores e a dos outros utentes. A mais evidente é a divergência sobre a tendência no cumprimento das regras do PMLS pelos pescadores. Na óptica maioritária dos outros utentes houve uma melhoria do cumprimento nos últimos 4-5 anos (apesar de não significativa), que não é corroborada pela maioria dos pescadores (que atesta maioritariamente que a frequência do incumprimento das regras do PMLS ficaram inalteradas nos últimos 4-5 anos). A comparação das distribuições das duas respostas confirma uma diferença significativa de percepção $(W=796,5 ; p=0,036)$, o que indica uma estranha incoerência entre actores e observadores do mesmo fenómeno (com os últimos a ser mais optimistas ou benevolentes).

\section{Conclusões}

Considerando o inquérito aos utentes do PMLS na globalidade, sobressai uma visão positiva para a evolução do PMLS e da sua interacção com a pesca no período de 2007-2008 a 2012, com a excepção de alguns aspectos socioeconómicos importantes ligados à pesca:

$>\mathrm{O}$ número de respostas a apontar para alguma melhoria é mais que o dobro das que apontam para alguma deterioração, num ratio global de 2.04:1. Dos 37 indicadores de tendência, a moda está situada numa percepção de melhoria em 20 e de deterioração em dois deles (sendo os resultados significativos para o tamanho da amostra em cinco positivos e um negativo);

$>$ Existe um optimismo generalizado nos indicadores ecológicos (oito dos 11 indicadores com modas em percepções de alguma melhoria, dois deles significativos) e mais moderado nos indicadores de governança (oito dos 15 indicadores com modas positivas, um deles significativo), não havendo nenhum indicador ecológico ou de governança com moda negativa;

$>$ Para os indicadores socioeconómicos existe uma apreciação menos uniforme entre assuntos, havendo percepções globalmente negativas para alguns indicadores ligados directamente aos pescadores (dois, ambos significativos) e percepções globalmente positivas para indicadores mais relacionados com outros utentes ou a comunidade geral (quatro, dos quais dois significativos). Esta separação entre pescadores e outros utentes é também evidente na comparação da distribuição de respostas em perguntas idênticas para os dois grupos, onde se detectam dife- renças significativas na evolução dos rendimentos e no encontro de soluções de gestão (em ambos os casos com percepções melhores para as outras actividades que para a pesca no PMLS).

Estes resultados estão genericamente consonantes com os resultados apresentados por Batista et al., (2011) que, com base em avaliação subjectiva de peritos antes $\mathrm{e}$ depois da implementação do POPNA, consideraram que houve uma melhoria para indicadores ecológicos e de governança e uma deterioração para indicadores sociais e económicos.

Comparando os resultados do presente estudo com os resultados do inquérito para os pescadores do PMLS (Stratoudakis et al., 2015), denota-se uma divergência prevalecente nos assuntos ecológicos e uma convergência nos assuntos socioeconómicos e de governança:

$>$ Existem diferenças significativas nas respostas para a maioria dos indicadores ecológicos de tendência comuns nos dois inquéritos, com os outros utentes a serem significativamente mais positivos que os pescadores do PMLS na percepção de evolução da abundância das espécies com interesse comercial, da abundância dos juvenis e de grandes peixes e no número total de espécies. Para algumas destas variáveis existe já alguma literatura a apoiar as percepções de melhoria (Cunha et al., 2014; Henriques et al. 2013; Horta e Costa et al. 2013). Todavia não é possível com o presente estudo avaliar se a discrepância aqui observada resulta de diferenças objectivas no sistema de referências dos dois grupos em termos de áreas de observação e espécies focais (e.g. Yasué et al., 2010; Leleu et al., 2012) ou diferenças subjectivas resultantes de enviesamento nas heurísticas utilizadas (e.g. Davis et al. 2004; Tversky and Kahneman, 1974).

$>$ A principal convergência ecológica prende-se com a importância das macroalgas no ecossistema costeiro local e no papel da "poluição" no seu desaparecimento repentino (uma narrativa quase idêntica entre inquiridos com mais que 30 anos de experiência no local nas várias categorias de utentes). Existe também alguma convergência na percepção do aumento de espécies raras ou estranhas, corroborada também por literatura que salienta o papel da zona de Arrábida como fronteira entre comunidades de zonas biogeográficas distintas (Henriques et al., 2007) e o aumento do aparecimento de exemplares de ecossistemas mais tropicais (Horta e Costa and Gonçalves, 2013);

$>$ Nos indicadores socioeconómicos e de governança as comparações são indirectas (porque maioritariamente têm de ser conjugadas respostas para indicadores de tendência dos utentes com respostas para indicadores de estado dos pescadores), mas no 
geral prevalece uma visão convergente, tanto sobre aspectos positivos (fortalecimento do papel da Associação, aumento do conhecimento sobre o PMLS) ou neutros (falta de agravamento da relação com a entidade gestora do PMLS), como sobre aspectos negativos (impactos da exclusão de pescadores do PMLS, divergência de rendimentos de pescadores com outros utentes e pessimismo sobre a atractividade da pesca para os jovens);

$>$ A principal divergência não ecológica surge na percepção do cumprimento das regras do PMLS pelos pescadores, onde - paradoxalmente - os outros utentes têm uma visão significativamente mais optimista que os próprios pescadores do PMLS.

Apesar do esforço para desenhar o painel de inquiridos de forma equitativa entre categorias e procurando metodologias ajustadas na realidade local para encontrar números suficientes de informantes adequados (Davis and Wagner, 2003), a implementação deste estudo enfrentou algumas dificuldades que não foram previstas:

> Apesar do número de inquiridos por categoria de utentes ter sido semelhante ao apresentado num estudo recentemente publicado seguindo a mesma metodologia (Pajaro et al., 2010), a dificuldade de encontrar pescadores profissionais a operar fora do PMLS mas com conhecimento sobre a situação actual do Parque, condicionou o número total de inquiridos e possivelmente limitou a capacidade de detectar mais resultados significativos entre os indicadores com modas a sugerir mudança;

$>$ A construção do questionário dos pescadores usando perguntas ligadas com a sua actividade diminuiu a comparabilidade com o questionário para os outros utentes, tanto porque em algumas perguntas o enunciado não é idêntico (e.g. abundância de peixes juvenis não é idêntico com peixes capturados abaixo do tamanho mínimo legal) como porque no questionário dos pescadores alguns indicadores de tendência só surgirão indirectamente após a repetição do inquérito e a obtenção de novas respostas sobre indicadores de estado;

A aceitação pessoal do PMLS (ultima pergunta no inquérito) mostrou-se correlacionada com o ratio de respostas positivas/negativas na totalidade do inquérito (Fig. 1b) e inversamente correlacionada com os anos de convivência com o sistema (Fig. 1a). Este padrão pode estar influenciado pela opinião mais negativa de pescadores que operam fora do PMLS (que tinham geralmente mais anos de exposição no sistema), mas não se limita a isto: observou-se também nos inquéritos efectuados em Lira e Cedeira aonde a definição dos pescadores incluídos foi menos conflituosa (Vidal e Verisimo, comunicação pessoal) e foi também relatado na literatura (percepções positivas sobre AMP inver- samente relacionadas com a senioridade do pescador Leleu et al., 2012). Independentemente do motivo para o aparecimento deste padrão, o seu efeito foi testado na análise dos dados, identificando indicadores para os quais a distribuição das respostas de "optimistas" e restantes eram divergentes.

\section{Appendix}

Supporting Information associated with this article is available on-line at http://www.aprh.pt/rgci/pdf/rgci-572_Stratoudakis_Supporting-Information.pdf

\section{Agradecimentos}

Este trabalho teve o apoio e colaboração de um elevado número de pessoas. Devemos cronologicamente destacar:

- A AAPCS (através dos seus presidentes Arsénio Caetano e António Pila e a sua secretária Carina Reis) pelo acompanhamento geral do projecto MAIA e o apoio operacional deste estudo;

- Juan Freire, Inma Alvarez e Nuria Fernandez (Universidade de Corunha) e Cristina Pita (Universidade de Aveiro) pelo contributo na realização do workshop que levou ao desenvolvimento da metodologia;

- Antonio Marques e Tiago Cagica (Câmara Municipal de Sesimbra) pelo envolvimento na identificação de utentes e o fornecimento de apoio logístico para a realização de entrevistas;

- Lia Vasconcelos e o projecto MARGOV por fornecer a base participativa para conhecer muitos dos utentes contactados neste estudo;

- Duarte Vidal e Patricia Verisimo (Universidade de Corunha) pelas informações sobre a aplicação do mesmo questionário nas AMPs de Cedeira e Lira na Galiza;

- Paulo Oliveira (IPMA) pela leitura crítica do manuscrito.

Este trabalho foi desenvolvido no âmbito do projecto MAIA (INTERREG IVB-Espaço Atlântico, 2009-1/143).

\section{References}

Batista, M.I.; Baeta, F.; Costa, M.J.; Cabral, H.N. (2011) - MPA as management tools for small-scale fisheries: the case-study of Arrábida Marine Protected Area (Portugal). Ocean and Coastal Management, 54(2):137-147. DOI: 10.1016/j.ocecoaman. 2010.10.032

Cabral, H.N.; Batista M.; Baeta, F.; Alves, A.; Costa, M.J. (2008) Avaliação do impacto das condicionantes na Área Marinha do Parque Natural de Arrábida à actividade da pesca comercial e lúdica, a náutica de recreio e ao mergulho. Relatório Final. Instituto de Oceanografia, FCUL, Lisboa. $242 \mathrm{pp}+$ anexos. Não publicado.

Carneiro, G. (2011) - The Luiz Saldanha Marine Park: an overview of conflicting perceptions. Conservation and Society, 9(4): 325333. DOI: $10.4103 / 0972-4923.92149$

Cunha, A.H., Erzini, K., Serrão, E.A., Gonçalves, E., Borges, R., Henriques, M., Henriques, V., Guerra, M., Duarte, C., Marbá, N., Fonseca, M. (2014) - Biomares, a LIFE Project to restore and manage the biodiversity of Prof. Luiz Saldanha Marine Park. Journal of Coastal Conservation. 18(6):643-655. DOI: 10.1007/s11852-014-0336-x

Davis, A.; Wagner, J.R. (2003) - Who knows? On the importance of identifying "experts" when researching local ecological knowledge. Human Ecology, 31(3):463-489.

Davis, A.; Hanson, J.M.; Watts, H.; MacPherson H. (2004) - Local ecological knowledge and marine fisheries research: the case of white hake (Urophycis tenuis) predation on juvenile American 
lobster (Homarus americanus). Canadian Journal of Fisheries and Aquatic Sciences, 61(7):1191-1201. DOI: 10.1139/F04-070

Henriques, M.; Gonçalves, E.J.; Almada, V.C. (2007) - Rapid shifts in a marine fish assemblage follow fluctuations in winter sea conditions. Marine Ecology Progress Series, 340:259-270.

Henriques, S.; Pais, M.P.; Costa, M.J.; Cabral, H.N. (2013) - Seasonal variability of rocky fish assemblages: detecting functional and structural changes due to fishing effort. Journal of Sea Research, 79:50-59. DOI: 10.1016/j.seares.2013.02.004

Henriques, V.; Guerra, M.T.; Mendes, B.; Gaudêncio, M.J., Fonseca, P. (in press) - Benthic habitat mapping in a Portuguese marine protected area using EUNIS: an integrated approach. Journal of Sea Research. DOI: 10.1016/j.seares.2014.10.007

Horta e Costa, B.; Gonçalves, E. (2013) - First occurrence of the Monrovia doctorfish Acanthurus monroviae (Perciformes: Acanthuriade) in European Atlantic waters. Marine Biodiversity Records, 6:e20. DOI: 10.1017/S1755267213000055

Horta e Costa, B.; Erzini, K.; Caselle, J.E.; Folhas, H.; Gonçalves, E. (2013) - "Reserve effect" within a temperate marine protected area in the North-eastern Atlantic (Arrábida Marine Park, Portugal). Marine Ecology Progress Series, 481:11-24. DOI: 10.3354/meps 10204

Leleu, K., Alban, F., Pelletier, D., Charbonnel, E., Letourneur, Y., Boudouresque, C.F. (2012) - Fishers' perceptions as indicators of the performance of Marine protected Areas (MPAs). Marine Policy 36(2):414-422. DOI: 10.1016/j.marpol.2011.06.002

Norman, G. (2010) - Likert scales, levels of measurement and the "laws" of statistics. Advances in Health Science Education, 15(5):625-632. DOI: 10.1007/s10459-010-9222-y

Pajaro, M.G.; Mulrennan, M.E.; Alder, J.; Vincent, A.C.J. (2010) Developing MPA effectiveness indicators: comparison within and across stakeholder groups and communities. Coastal Management 38: 122-143. DOI: 10.1080/08920751003633094

Pomeroy, R.S.; Parks, J.; Watson, L. (2004) - How is your MPA doing? A guidebook of natural and social indicators for evaluating marine protected areas and management effectiveness. IUCN, Gland, Switzerland and Cambridge, UK. Xvi + 216 pp. ISBN: 2-8317-0735-8; 978-2-8317-0735-8. Available online at https://portals.iucn.org/library/node/8417

$\mathrm{R}$ Core Team (2014) - $R$ : A language and environment for statistical computing. R Foundation for Statistical Computing, Vienna, Austria. Available online at http://www.R-project.org/

Stratoudakis, Y.; Fernándes, F.; Henriques, M.; Martins, J.; Martins R. (2015) - Situação ecológica, socioeconómica e de governança após a implementação do primeiro plano de ordenamento no Parque Marinho professor Luiz Saldanha (Arrábida, Portugal): I - informações e opiniões de pescadores. Journal of Integrated Coastal Zone Management / Revista de Gestão Costeira Integrada, 15(2):153-166. DOI: 10.5894/rgci571

Tversky, A.; Kahneman, D. (1974) - Judgment under uncertainty: heuristics and biases. Science, 185(4157):1124-1131. DOI: 10.1126/science. 185.4157 .1124

Yasué, M.; Kaufman, L.; Vincent, A.C.J. (2010) - Assessing ecological changes in and around marine reserves using community perceptions and biological surveys. Aquatic Conservation: Marine and Freshwater Ecosystems, 20(4):407-418. DOI: 10.1002/aqc. 1090

Vasconcelos, L.; Caser, U.; Pereira, M.J.R.; Gonçalves, G.; Sá, R. (2012) - MARGOV - Building social sustainability. Journal of Coastal Conservation, 16(4):523-530. DOI: 10.1007/s11852012-0189-0 